\title{
The Acute Effects of Reserpine and NSD-1015 on the Brain Serotonin Synthesis Rate Measured by an Autoradiographic Method
}

\author{
Dorotea Mück-Šeler, Ph.D., and Mirko Diksic, Ph.D.
}

The rate of serotonin (5-HT) synthesis was measured in the discrete regions of the rat brain utilizing an autoradiographic method and $\alpha l^{14} \mathrm{Cl}$ methyl-L-tryptophan as a tracer after an acute treatment with reserpine (10 $m g / k g ~ I P)$ or NSD-1015 (m-hydroxybenzylhydrazine) $(100 \mathrm{mg} / \mathrm{kg} \mathrm{IP})$. Controls were injected with the same volume of solvent in place of reserpine or NSD-1015. Our results showed that reserpine induced a statistically significant (except for medial geniculate body) decrease in the rate of 5-HT synthesis in a large number of discrete brain structures. Reserpine had no influence on the plasma concentration of amino acids sharing the same carrier with tryptophan nor on the fraction of plasma-free tryptophan. NSD-1015 induced a statistically significant increase $(p<.05)$ in the rate of 5-HT synthesis in 20 out of 28 brain regions but produced a pronounced decrease in the rate of 5-HT synthesis in the pineal body. This decrease in the pineal body serotonin synthesis rate is most likely the result of the loss of the label in the form of 5-hydroxy-a $\left[{ }^{14} \mathrm{C}\right]$ methyl-L-tryptophan [5-OHMTrp] that is not metabolized further because aromatic amino acid decarboxylase was inhibited. The data showing that there was no loss of the 5-OHMTrp from other brain structures as result of reserpine are also given. NSD-1015 treatment also induced a time-dependent increase in the plasma concentration of free tryptophan that becomes significant 30 minutes after NSD-1015 injection. Our results suggest that reserpine induces a decrease in 5-HT synthesis probably via direct or indirect inhibition of tryptophan hydroxylase activity. Since NSD-1015 alone increased the rate of 5-HT synthesis, the measurement of 5-HT synthesis in previous experiments using NSD-1015 and measuring the rate of 5-hydroxytryptophan accumulation after NSD-1015 induced inhibition of decarboxylase activity should be interpreted with reservation.

[Neuropsychopharmacology 12:251-262, 1995]
KEY WORDS: Serotonin synthesis rate; Reserpine; NSD-1015; Rat brain; Autoradiography; $\alpha$-Methyl-tryptophan

From the Laboratory for Molecular Neuropharmacology (DMS), Ruder Bošković Institute, Zagreb, Croatia; Department of Neurology and Neurosurgery and Montreal Neurological Institute and Hospital (MD), Cone Neurosurgical Research Laboratory, McGill University, 3801 University Street, Montreal, Quebec, Canada.

Results were in part presented at the Twenty-Third Annual Meeting of Neuroscience Society in Washington, DC, November 7-12,
In the 1970s and the 1980s abundant investigations of the central serotoninergic system has led to the conclusion that serotonin (5-HT) plays a role in the regulation

1993 (abstract No. 126.18).

Address correspondence to: Mirko Diksic, Ph.D., Montreal Neurological Institute, 3801 University Street, Montreal, Quebec, Canada H3A $2 B 4$.

Received May 25, 1994; revised September 21, 1994; accepted October 14, 1994. 
of many physiological functions (sleep, food intake, mood) (Byerly and Risch 1985) as well as in the etiology of mental disorders (depression, schizophrenia) (Hrdina et al. 1993; Joyce 1993).

The synthesis of 5-HT in vivo occurs in a two-step enzymatic process. The first rate-limited step is 5-hydroxylation of the essential amino acid L-tryptophan catalyzed by tryptophan hydroxylase (TPH; EC 1.14.16.4), an enzyme located only in serotoninergic neurons (Kuhar et al. 1971). The product of this reaction, 5-hydroxytryptophan (5-HTP), is decarboxylated by a nonspecific enzyme, aromatic amino acid decarboxylase (EC 4.1.1.28) to 5-HT. A variety of methods have been used to measure the in vivo rate of 5 -HT synthesis. In general, previous methods have required some form of pharmacological manipulation. This means that any potential feedback in the synthesis pathway can be, at least in part, influenced by the drug used and may thereby distort the results. On the other hand, the use of radiolabeled tryptophan has not proven very successful because of the extensive incorporation of this essential amino acid into proteins and of the very rapid loss of its metabolite from the brain.

In the present work we used a recently developed autoradiographic method (Diksic et al. 1990; Nagahiro et al. 1990) that permits the in vivo measurement of the rate of 5-HT synthesis with good anatomical resolution and without any pharmacological treatment except the one investigated. The method is based on the tracer principle, in which an analog of L-tryptophan [namely, $\alpha$-methyl-tryptophan ( $\alpha$-MTrp) labeled with ${ }^{14} \mathrm{C}$ or ${ }^{3} \mathrm{H}$ ] is used. This compound follows the metabolic pathway of tryptophan, being converted into radioactively labeled $\alpha$-methyl-serotonin ( $\alpha$-M5-HT). Unlike 5-HT, $\alpha-\mathrm{M} 5-\mathrm{HT}$ is not a substrate for monoamine oxidase.

Within the neuron 5-HT is stored in at least two compartments: a large vesicular pool and a much smaller cytoplasmic pool (Kleven et al. 1983; Kuhn et al. 1985). The vesicular transport system consists of a vesicular ATP-driven $\mathrm{H}^{+}$pump that acidifies the organelle interior and a vesicular amine transporter that exchanges these internal $\mathrm{H}^{+}$ions with cytoplasmic 5-HT (Rudnick and Clark 1993). The transport of 5-HT into vesicles can be almost completely prevented by irreversible binding of reserpine (Schuldiner et al. 1993). This inhibitory effect of reserpine results in a significant 5-HT release and a decrease in its levels in the brain tissue with a simultaneous increase in 5-HIAA levels (Halaris and Freedman 1975). There are contradictory reports about reserpine effects on 5-HT synthesis. Both an increase (Hjorth 1992) and an unchanged (Long et al. 1983) accumulation of 5-HTP were observed after inhibition of decarboxylase activity with NSD-1015 and RO4/4501/1, respectively. On the other hand, to our knowledge, there are no data on the effects of NSD1015 alone on the rate of 5-HT synthesis in the rat brain.
Since these drugs can affect the steady state of plasma tryptophan as well as brain 5-HT synthesis (present data), their influence on 5-HT synthesis must be known before we can evaluate any other drug. Consequently, the aim of the present work was to determine, using the autoradiographic method, whether an acute administration of reserpine alone or of NSD-1015 alone can have an influence on the rate of brain 5-HT synthesis.

\section{MATERIAL AND METHODS}

\section{Animals}

Sprague-Dawley male rats (Charles River, NC) weighing between 220 and $250 \mathrm{~g}$ were used in this study. Rats were housed in our animal facility (room temperature $22^{\circ} \mathrm{C}$; 12-hour day-night cycle) for at least 3 days before being used in experiments. Before experiments the animals were fasted overnight with water supply ad libitum. All rats were sacrificed between 2:00 P.M. and 4:00 P.M. The mean physiological parameters $(\mathrm{pH}$, $\mathrm{pCO}_{2}, \mathrm{pO}_{2}$, haematocrit, body weight) for all experimental groups were within the range of previously published values in saline-treated rats (Nagahiro et al. 1990).

\section{Experimental Procedure}

Under light halothane (0.5-1\%) anesthesia, plastic catheters were inserted into femoral arteries (for blood sampling) and veins (for tracer injection). Immediately after surgical treatment, animals were injected intraperitoneally (IP) with $10 \mathrm{mg} / \mathrm{kg}$ of reserpine. The control groups were injected with the same volume of solvent instead of reserpine (water with a few drops of glacial acetic acid). Rats were placed in loose-fitting plaster casts and then allowed to wake up. NSD-1015 (100 $\mathrm{mg} / \mathrm{kg}$, dissolved in saline) or saline (control) were administered by IP injection one hour and a half after surgical treatment. Two hours after reserpine or 30 minutes after NSD-1015 application, $50 \mu \mathrm{Ci}$ of $\left[{ }^{14} \mathrm{C}\right]-\alpha$-MTrp was injected IV in $1 \mathrm{ml}$ of saline over 2 minutes, using constant infusion with an injection pump. With the beginning of the tracer injection, arterial blood samples were taken at progressively increased time intervals until killing time. The blood samples were centrifuged for 3 minutes at $12,500 \mathrm{~g}$. Twenty microliters of plasma were deproteinized with $10 \mu \mathrm{l}$ of $20 \%$ trichloroacetic acid (TCA). After mixing and spinning ( 2 minutes at $12,500 \mathrm{~g}), 20 \mu \mathrm{l}$ of supernatant was measured for plasma $\left[{ }^{14} \mathrm{C}\right]$ radioactivity (input function) by liquid scintillation counting. Animals were killed by guillotine one or two and half hours after tracer injection. The brains were removed, frozen in freon, and cut into $30-\mu \mathrm{m}$ slices in a cryostat at about $-20^{\circ} \mathrm{C}$. Brain sections were mounted on glass slides and exposed to $X$-ray film along 
with ${ }^{14} \mathrm{C}$-polymer standards for 3 weeks in order to obtain autoradiograms. The films were developed and radioactivity concentrations in different structures determined using a microcomputer-based image analyzing system (Image Calculator; Soquelec Ltd., Montreal) consisting of a video camera, a frame grabber, and an IBM AT compatible computer and appropriate software.

In a separate set of experiments the effect of acutely administered reserpine was evaluated for a possible loss of the label from the tissue in reserpinized rats. Thirty micro curies inches of $\alpha-\left[{ }^{14} \mathrm{C}\right] \mathrm{MTr} p$ were injected IV 2 hours after arterial and venous catheters were implanted. One hundred minutes after the tracer injection rats were injected with reserpine $(10 \mathrm{mg} / \mathrm{kg}$; four rats), and another group of four rats were injected with the solvent for reserpine (considered as controls). The rats were killed 100 minutes and 200 minutes after reserpine and tracer injections, respectively. The arterial blood samples were collected soon after tracer injection, as described.

\section{Determination of Plasma LNAA Concentrations}

At the beginning, midpoint, and end of each experiment, a further $50 \mu$ l of plasma was deproteinized with $25 \mu \mathrm{l}$ of $20 \%$ TCA. After centrifugation, $50 \mu \mathrm{l}$ of supernatant was stored at $-70^{\circ} \mathrm{C}$ until analyzed for the large neutral amino acids (LNAA; valine, isoleucine, leucine, phenylalanine, tyrosine, total tryptophan). The fraction of non-albumin-bound (free) tryptophan in the plasma was measured by filtering $50 \mu \mathrm{l}$ of plasma through a Gelman Sciences NanoSpin ${ }^{\mathrm{TM}} 10,000 \mathrm{MWCO}$ filter, spinning at $14,000 \mathrm{rpm}$ for 10 minutes. Total and free tryptophan and other LNAA concentrations were measured by the HPLC method (Takada et al. 1993) using a postcolumn o-phtalaldehyde derivatization.

In a separate set of experiments, the influence of NSD-1015 on the plasma tryptophan concentration was evaluated. Rats were implanted with a plastic catheter in the femoral artery and placed in loose-fitted plaster casts. Two hours after surgical treatment, the first blood sample was taken for the determination of total and free plasma tryptophan concentration. Following this, a group of nine animals received NSD-1015 $(100 \mathrm{mg} / \mathrm{kg}$ IP), while eight animals (control group) were treated with an IP injection of saline. Arterial blood samples were collected at $15,30,45,60,75,90,120$, and 150 minutes after injection of NSD-1015 or saline. The concentration of plasma total and free tryptophan was determined as described.

\section{Calculation of 5-HT Synthesis Rate}

The estimation of the rate of 5-HT synthesis in the brain using $\alpha-{ }^{14} \mathrm{C}-\mathrm{MTrp}$ is based on a three-compartment biological model: plasma, precursor, and metabolic (which are not necessarily distinct anatomical or tissue compartments) (Diksic et al. 1991; Nagahiro et al. 1990). Since the tracer (actually its metabolite) is irreversibly trapped in the brain, a simplified approach in the calculation of the synthesis rate can be used (Nagahiro et al. 1990). After an apparent steady state is reached, the distribution volume (DV) of tracer between plasma and tissue is a linear function of the exposure time $\Theta$. The slope of this linear function is equal to the constant for the unidirectional trapping of the tracer, or $K^{*}(\mathrm{ml} / \mathrm{g} /$ minute). This can be mathematically represented as:

$$
\begin{gathered}
\text { DV }=\Theta \cdot K^{*}+V_{\text {app }} \\
\Theta=\int_{0}^{\mathrm{T}} C_{p}(t) \cdot d t / C_{p}^{*}(T),
\end{gathered}
$$

where $C_{p}(t)[\mathrm{nCi} / \mathrm{g}]$ is the plasma tracer concentration as a function of time and $C_{p}{ }^{*}(T)[\mathrm{nCi} / \mathrm{g}]$ is tracer concentration in plasma at time $T$ and $V_{\text {app }}[\mathrm{ml} / \mathrm{g}]$ is the apparent volume of distribution of the tracer. The rate of 5 -HT synthesis $(R)$ was calculated as:

$$
R=C_{p} \cdot K^{\star} / L C \text {. }
$$

$C_{p}[\mathrm{nmol} / \mathrm{g}]$ is the concentration of the plasma nonprotein-bound tryptophan. The "lumped constant" $(L C)$ was found to be uniform throughout the brain having an average value of $0.42 \pm 0.07$ (Vanier et al. 1995).

\section{Statistics}

The statistical significance of the results was evaluated by the two-tailed $t$-test or by analysis of variance (ANOVA), followed by the Newman-Keuls's multiple comparison test.

\section{Drugs}

Reserpine (Sigma Chemical Co., St. Louis, MO) was dissolved in a few drops of glacial acetic acid and diluted with water ( $\mathrm{pH}$ about 3.5). NSD-1015 (m-hydroxybenzyl-hydrazine dihydrochloride, Sigma Chemical Co., St. Louis, MO) was dissolved in saline. Reserpine and NSD-1015 were prepared freshly before injections. $\alpha-\left[{ }^{14} \mathrm{C}\right]$ Methyl-L-tryptophan (specific activity of about $55 \mathrm{mCi} / \mathrm{mmol}$ ) was synthesized by us using the procedure described by Mzengeza et al. (1993).

\section{RESULTS}

\section{Effect of Reserpine on the Rate of 5-HT Synthesis}

A set of representative autoradiograms exemplifying brain distribution of tracer in control and reserpinetreated rats is shown in Figure 1. A visual inspection of these images confirms that the greatest uptake of the tracer was in the structures with a high concentration of serotoninergic cell bodies (dorsal and median raphe 


\section{Control}
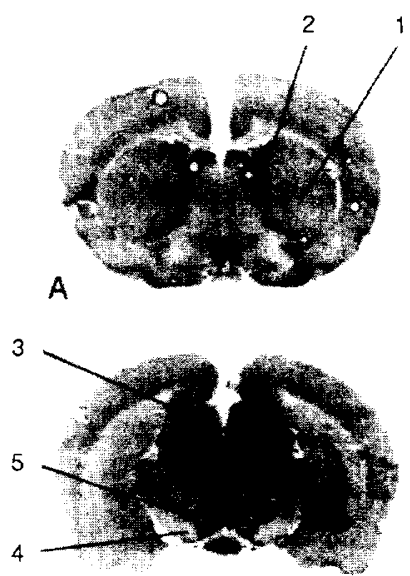

B

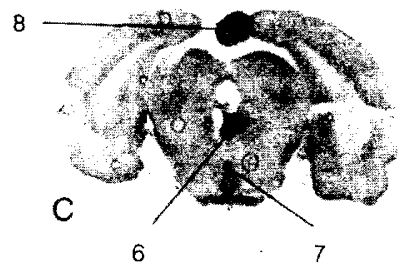

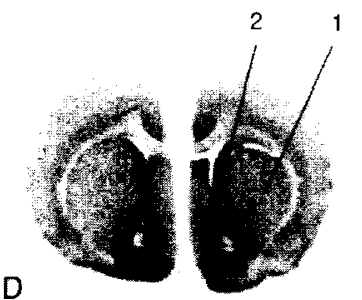

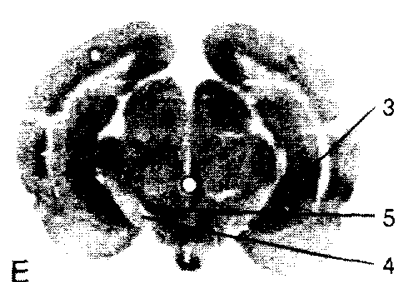

$E$

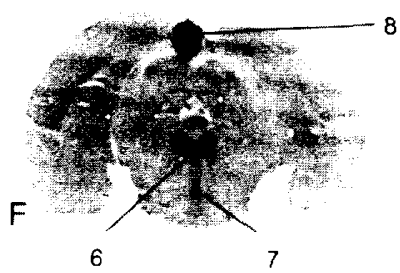

Reserpine

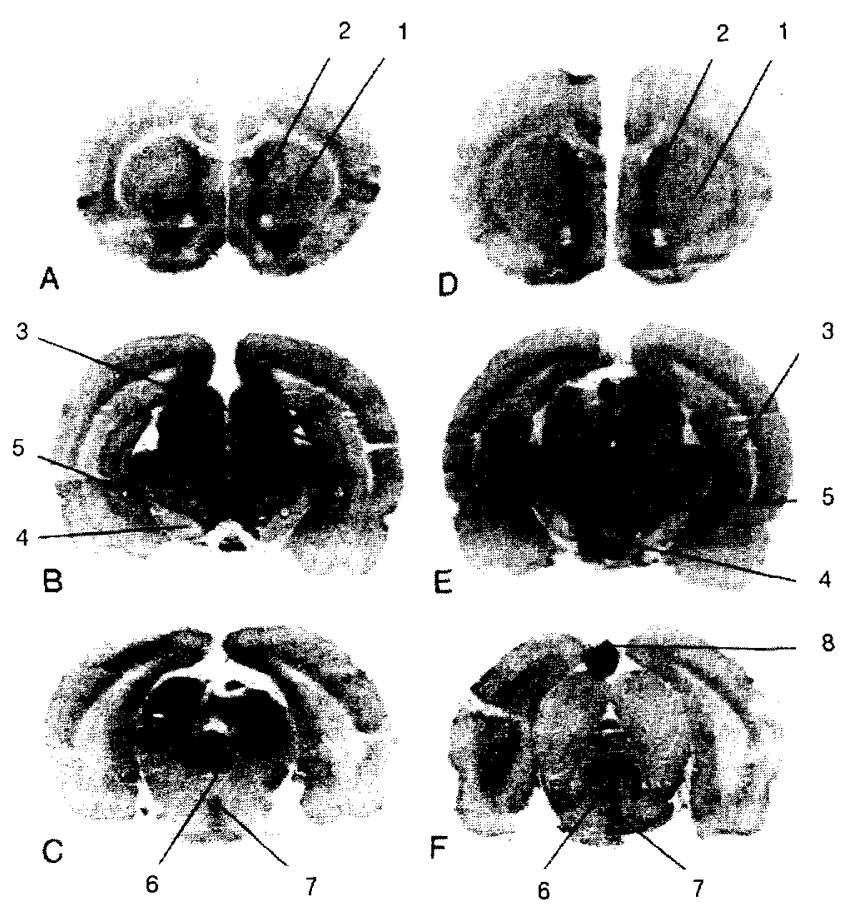

Figure 1. A representative set of autoradiograms obtained in control (left panels) and reserpine-treated (right panels) rats. Fifty $\mu \mathrm{Ci}$ of ${ }^{14} \mathrm{C}-\alpha$-MTrp was injected and rats were killed $60(\mathrm{~A}-\mathrm{C})$ and $150(\mathrm{D}-\mathrm{F})$ minutes after tracer injection. Some structures clearly visualized are (1) caudate nucleus lateral, (2) caudate nucleus medial, (3) hippocampus, (4) substantia nigra (pars reticulata), (5) substantia nigra (pars compacta), (6) dorsal raphe nucleus, (7) median raphe nucleus, (8) pineal body.

nuclei). There was also a nonuniform uptake of tracer in some brain structures. For example, a higher uptake of the tracer was seen in the medial part of the caudate, the substantia nigra, and a deep layer of the cortex. The tissue radioactivity was determined separately in the structures of the left and right hemispheres. Since there were no statistically significant differences found between the two sides, the values were averaged.

The relationship between distribution volume (DV) and exposure time $(\Theta)$ in several brain regions in control and reserpine-treated rats is shown in Figure 2. The slope of this linear relation is $K^{*}$, the parameter required for the calculation of the 5-HT synthesis rate (for details see Material and Methods). There was no significant difference in the plasma concentration of the LNAA between control and reserpine-treated animals (data not shown).

In Table 1 the rates of 5-HT synthesis for control and reserpine-treated rats in selected brain regions and in the pineal body are given. A decrease in the rate of synthesis is also expressed as "Difference (\%)" calculated as a percentage of the rates of synthesis in the control group. A decrease in the synthesis varied between $21 \%$ (medial geniculate body) and $55 \%$ (pineal body). A graphic comparison of the synthesis rates for the re- maining 15 brain structures is shown in Figure 3. A statistically significant decrease in the rate of 5-HT synthesis was observed in all brain structures of reserpinetreated rats except in the medial geniculate body, where a decrease was present but not significant.

Administration of reserpine 100 minutes after the beginning of tracer injection and sacrificing animals 100 minutes after reserpine did not produce a statistically significant ( $p>.05$; two tail $t$-test) difference in the distribution volume of the radioactively labeled tracer in 16 brain structures examined in the reserpine-treated rats as compared to the controls. The percent difference (calculated as \% distribution volume in the control group) varied from $-19 \%$ (medial raphe nucleus) to $5 \%$ (dorsal thalamus) in the structures examined. However, when one of the rats from the reserpine-treated group was removed because, for reasons unknown, its volume of distribution was substantially lower than the volumes of distribution in other rats (on statistical grounds it could be removed from the group as an outlier), the difference between the two groups became even smaller $(-11 \%$ median dorsal raphe to $5 \%$ caudate nucleus lateral part). A relative comparison of the DV in the two groups of rats (both with inclusion and exclusion of this outlier) is given in Figure 4. 
Dorsal Raphe

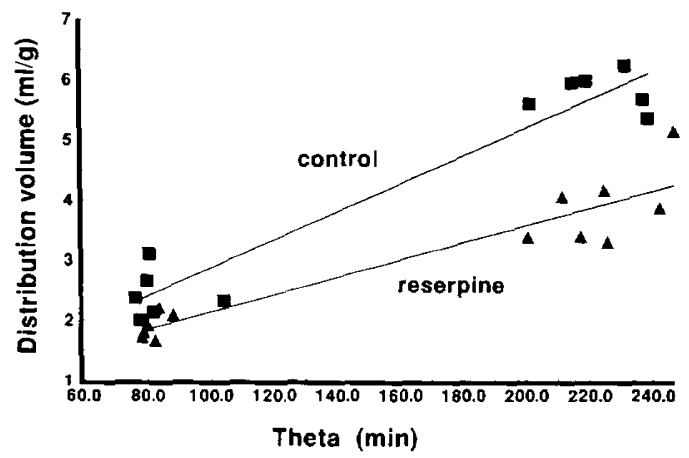

Hypothalamus

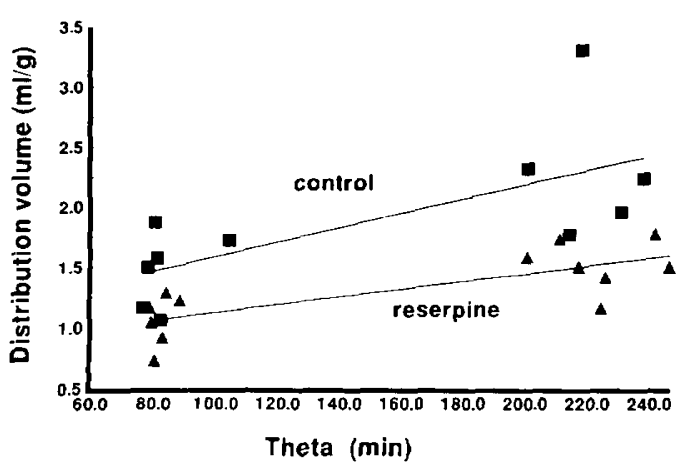

Median Raphe

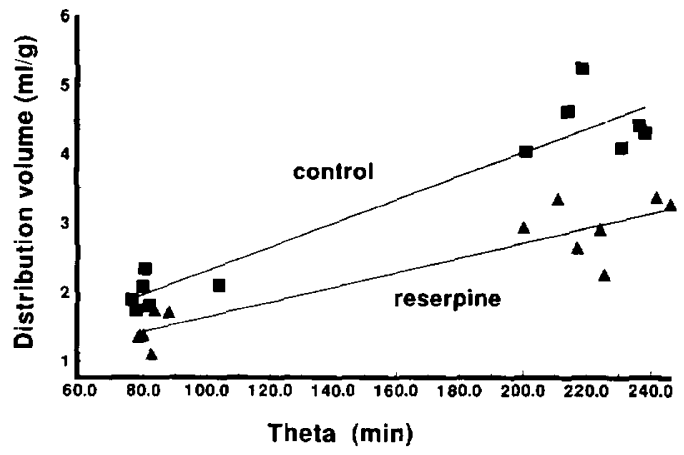

Figure 2. Representative plots of the tissue distribution volume of tracer for control $(\mathbf{\square})$ and reserpine-treated rats $(\mathbf{A})(\mathrm{killed}$ at 60 and 150 minutes after tracer injection) as a function of theta in some brain regions.

Table 1. The Rate of 5-HT Synthesis $\left(R ; \mathrm{pmol} \mathrm{g}^{-1}\right.$ minute $\left.^{-1}\right)$ in Representative Brain Structures and Pineal Body in Control and Reserpine-Treated Rats

\begin{tabular}{lrcr}
\hline \multicolumn{1}{c}{ Structure } & \multicolumn{3}{c}{$\mathbf{R}^{a}\left(\mathbf{p m o l} \mathbf{g}^{-\mathbf{1}}\right.$ minute $\left.^{-\mathbf{1}}\right)$} \\
\cline { 2 - 4 } & $\begin{array}{r}\text { Control } \\
(\boldsymbol{n}=\mathbf{1 2})\end{array}$ & $\begin{array}{c}\text { Reserpine } \\
(\boldsymbol{n}=\mathbf{1 3})\end{array}$ & $\begin{array}{c}\text { Difference } \\
(\mathbf{\%})^{b}\end{array}$ \\
\hline Dorsal raphe nucleus & $193 \pm 16$ & $119 \pm 13$ & $38^{c}$ \\
Medan raphe nucleus & $140 \pm 14$ & $87 \pm 11$ & $38^{c}$ \\
Hypothalamus & $49 \pm 16$ & $26 \pm 7$ & $47^{c}$ \\
Visual cortex & $48 \pm 14$ & $33 \pm 8$ & $31^{d}$ \\
Caudate nucleus lateral & $52 \pm 18$ & $28 \pm 7$ & $46^{c}$ \\
Caudate nucleus medial & $66 \pm 18$ & $35 \pm 10$ & $47^{c}$ \\
Substantia nigra pars reticulata & $31 \pm 11$ & $21 \pm 5$ & $32^{d}$ \\
Substantia nigra pars compacta & $58 \pm 16$ & $33 \pm 7$ & $43^{c}$ \\
Hippocampus ventral & $69 \pm 17$ & $48 \pm 9$ & $30^{c}$ \\
Medial geniculate body & $56 \pm 18$ & $44 \pm 10$ & 21 \\
Thalamus dorsal & $58 \pm 17$ & $41 \pm 15$ & $29^{c}$ \\
Pineal body & $342 \pm 102$ & $153 \pm 43$ & $55^{c}$ \\
\hline
\end{tabular}

"The rates are given as estimates $\pm S D ; n$ is the number of animals.

${ }^{b}$ Differences are given as a percentage reduction from the rate in control group. The difference is significant when reserpine group is compared to the control group.

${ }^{c} p<.001$ (two-tail $t$-test).

d $p<.01$ (two-tail $t$-test).

${ }^{e} p<.05$ (two-tail $t$-test). 


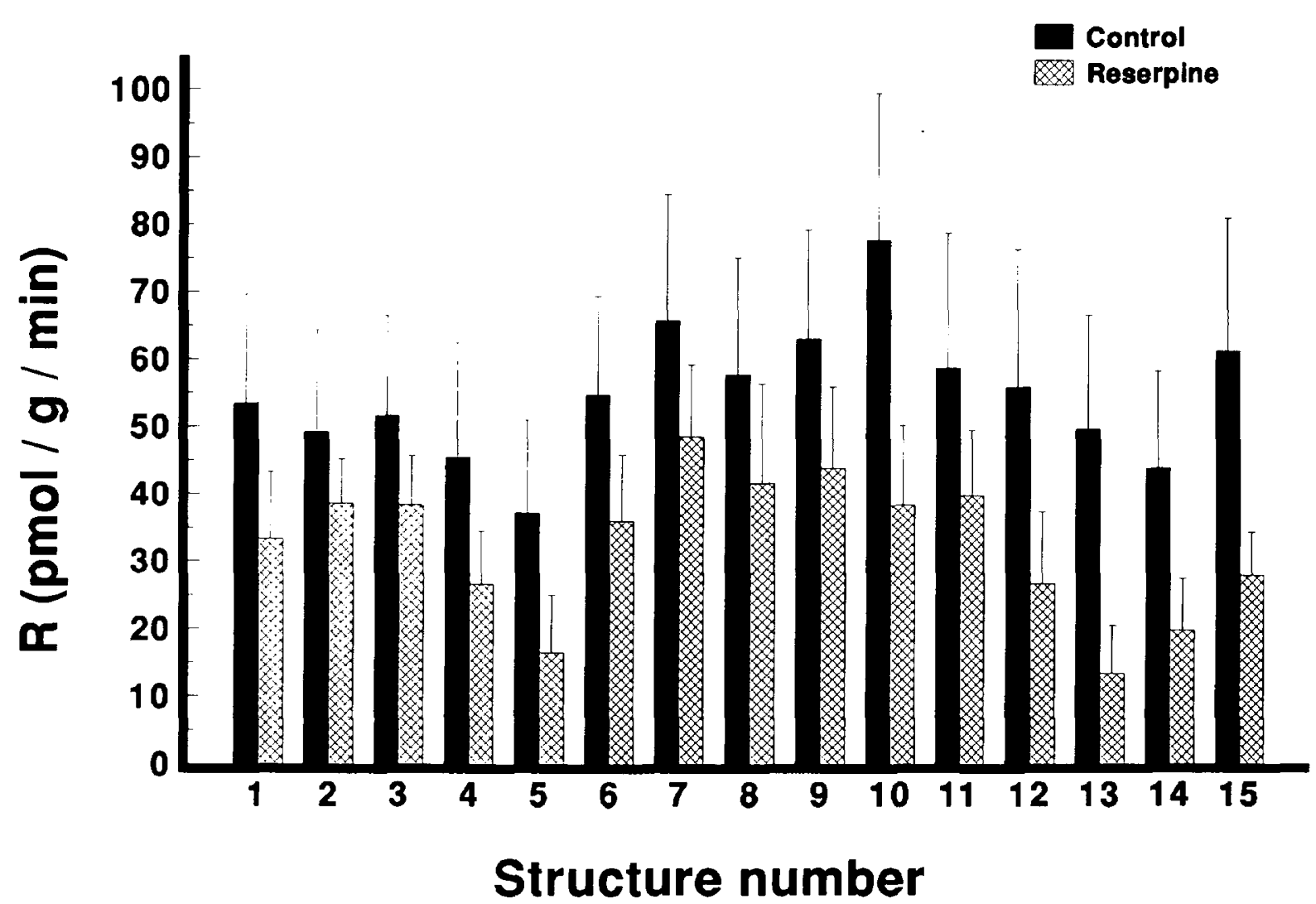

Figure 3. A comparison of the 5-HT synthesis rates $\left(\mathrm{R} ; \mathrm{pmol} \mathrm{g}^{-1} \mathrm{~min}^{-1}\right)$ in some brain structures (not given in Table 1) in control (filled bars) and reserpine-treated (crossed bars) rats. Each column represents mean $\pm \mathrm{SD}$ (shown as error bars) of 12 (control) or 13 (reserpine-treated) animals. The difference in $R$ between control and reserpine-treated rats is statistically significant ( $p<.05$ at least) in all regions. Structure numbers are: (1) raphe magnus, (2) ventral tegmental area, (3) medial forebrain bundle, (4) superior olive, (5) inferior colliculus, (6) superior colliculus, (7) lateral geniculate body, (8) ventral thalamus, (9) dorsal hippocampus, (10) nucleus accumbens, (11) auditory cortex, (12) parietal cortex, (13) sensory-motor cortex, (14) frontal cortex, (15) medial anterior olfactory nucleus.

\section{Effect of NSD-1015 on the Rate of 5-HT Synthesis}

The relationship between DV and exposure time $(\Theta)$ in the pineal body in control and NSD-1015-treated rats is shown in Figure 5. This structure was selected because of its profound decrease in the rate of 5-HT synthesis in contrast to increases in almost all other structures. NSD-1015 induced a nonuniform effect on the rate of 5-HT synthesis in rat brain regions (Table 2 and Figure 6). The increase varied between $13 \%$ (median raphe) to $94 \%$ (substantia nigra pars reticulata). A significant increase in the rate of 5-HT synthesis was observed in a large number of brain regions, while the rate of 5-HT synthesis was decreased in the inferior colliculus and was largely decreased in the pineal body (Table 2). There were also a few structures in which NSD-1015 did not have influence on the rate of 5-HT synthesis (Figure 6).

\section{Effect of NSD-1015 on Plasma Tryptophan Concentration}

NSD-1015 treatment induced time-dependent alterations in the plasma concentration of free tryptophan (Figure 7). Detailed statistical study (ANOVA, $F=2.5$, $\mathrm{df}=8,62, p<.05)$ showed that the increase in plasmafree tryptophan concentration was significant (ANOVA followed by Newman-Keuls, $p<.05$ ) only at 30 minutes after NSD-1015 injection when compared with the values before NSD-1015 injection. There were no changes in the concentration of plasma-free tryptophan in the control group of rats during the observed period (ANOVA, $F=1.55, \mathrm{df}=8,61, p>.05$ ) (Figure 7). The concentration of plasma total tryptophan during the experiment was stable in the control group (ANOVA, $F=$ $0.89, \mathrm{df}=2,23, p>.05$ ) as well as in the NSD1015-treated ( $F=0.08, \mathrm{df}=2,21, p>.05)$ group of animals. 

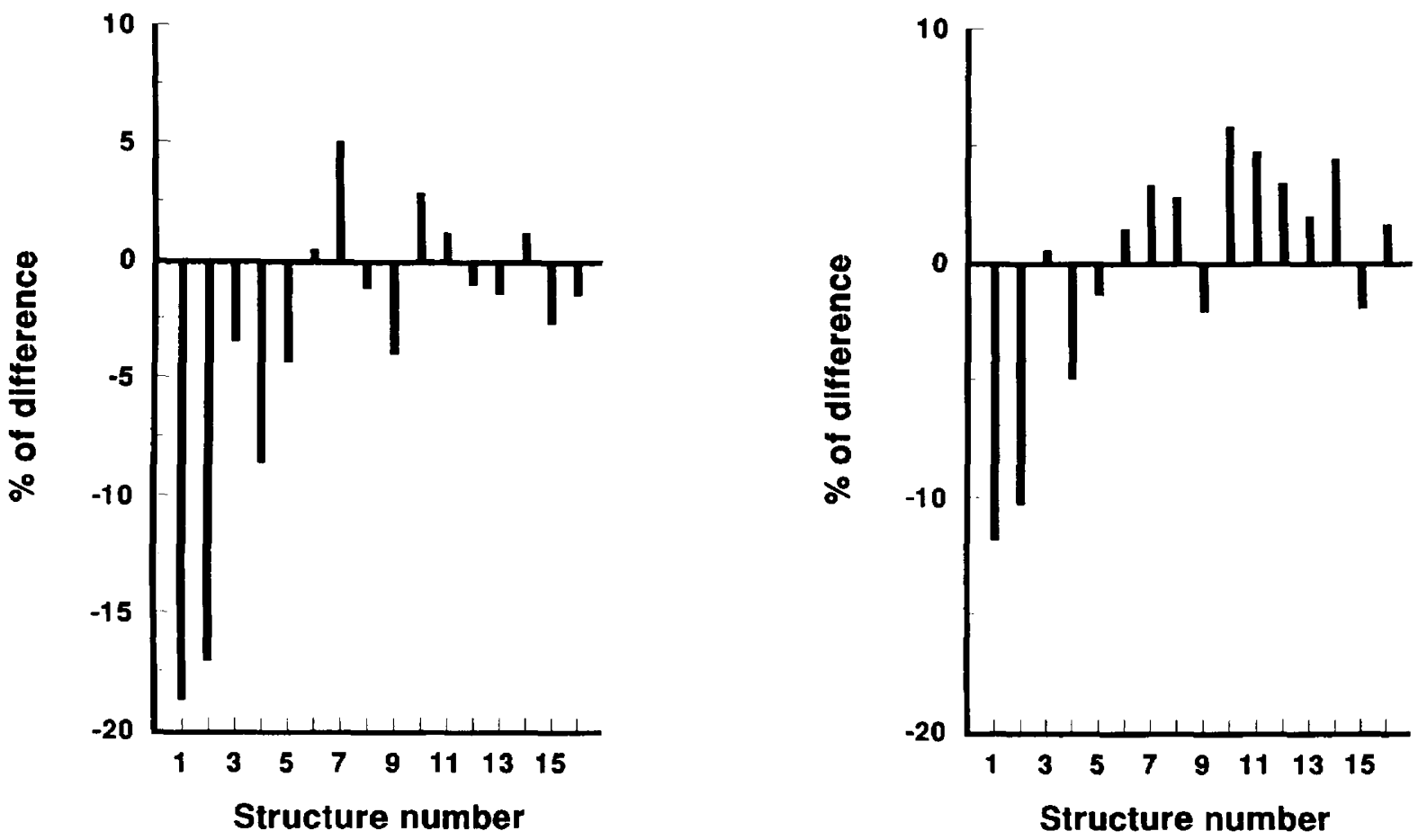

Figure 4. The effect of reserpine administration $(10 \mathrm{mg} / \mathrm{kg} ; 100$ minutes after the beginning of the tracer injection; killing rats 100 minutes after reserpine and 200 minutes after tracer injection) on the distribution volume of labeled $\alpha$-M5-HT in different brain regions is shown. The results are given as a percentage of difference of controls, calculated as the difference between the control and treated groups divided by the volume of distribution of the control group, and expressed as a percent. The left diagram shows the percent difference for all rats (four in each group), and the right graph shows the difference when a statistically significant outlier was removed. The structures corresponding to the numbers on the $x$ axis are (1) median raphe, (2) dorsal raphe, (3) ventral tegmental area, (4) pineal body, (5) hypothalamus, (6) ventral thalamus, (7) dorsal thalamus, (8) ventral hippocampus, (9) dorsal hippocampus, (10) caudate nucleus (lateral part), (11) caudate nucleus (medial part), (12) nucleus accumbens, (13) visual cortex, (14) auditory cortex, (15) parietal cortex, (16) sensory motor cortex.

\section{DISCUSSION}

In the present work we have found that reserpine and NSD-1015 produced opposite effects on the rate of 5-HT synthesis in the rat brain (Table 1 and Figures 3 and 6). Moreover, we have shown, for the first time and with good anatomical resolution, that an acute reserpine treatment induces a decrease in the rate of 5-HT synthesis (statistically significant in 26 out of 27 brain regions studied; Table 1 and Figure 3), while NSD-1015 induces an increase in the rate of 5 -HT synthesis in most structures (Figure 6). The pronounced decrease in 5-HT synthesis observed in the pineal body after NSD-1015 was most likely the results of the loss of 5-hydroxy- $\alpha$ methyl-L-tryptophan (5-OHMTrp), since decarboxylase inhibition prevents the conversion into $\alpha-\mathrm{M} 5-\mathrm{HT}$ and its subsequent storage into vesicles. In addition, the pineal body does not have a blood-brain barrier (BBB), thus allowing even easier loss of this labeled intermediary from this structure. The values of the regional 5-HT synthesis rate in both control groups of animals are similar to the values obtained earlier in saline-treated rats using the same autoradiographic method (Nagahiro et al. 1990).

Our findings on the influence of reserpine on the rate of 5-HT synthesis are in contradiction with the recently published data of Hjorth (1992) who found an increase in the rate of 5-HT synthesis after an acute reserpine application. Since there are some experimental differences between these two investigations, a direct comparison of results is not possible. The most important difference between our and Hjorth's (1992) measurements is that he estimated the rate of $5-\mathrm{HT}$ synthesis by measuring 5-HTP accumulation after inhibition of aromatic amino acids decarboxylase with NSD1015 (the influence of NSD-1015 on 5-HT synthesis will be discussed later). Further, the animals were sacrificed $11 / 2$ hours after reserpine administration and the rate of 5-HT synthesis was determined only in limbic, striatal, and cortical brain areas using surgical tissue sampling. In the present work we used a higher dose of reserpine (10 $\mathrm{mg} / \mathrm{kg} \mathrm{IP}$ ) as compared with $5 \mathrm{mg} / \mathrm{kg}$ used by Hjorth (1992) in an attempt to avoid "partially reserpinized" animals (Halaris and Freedman 1975) during 


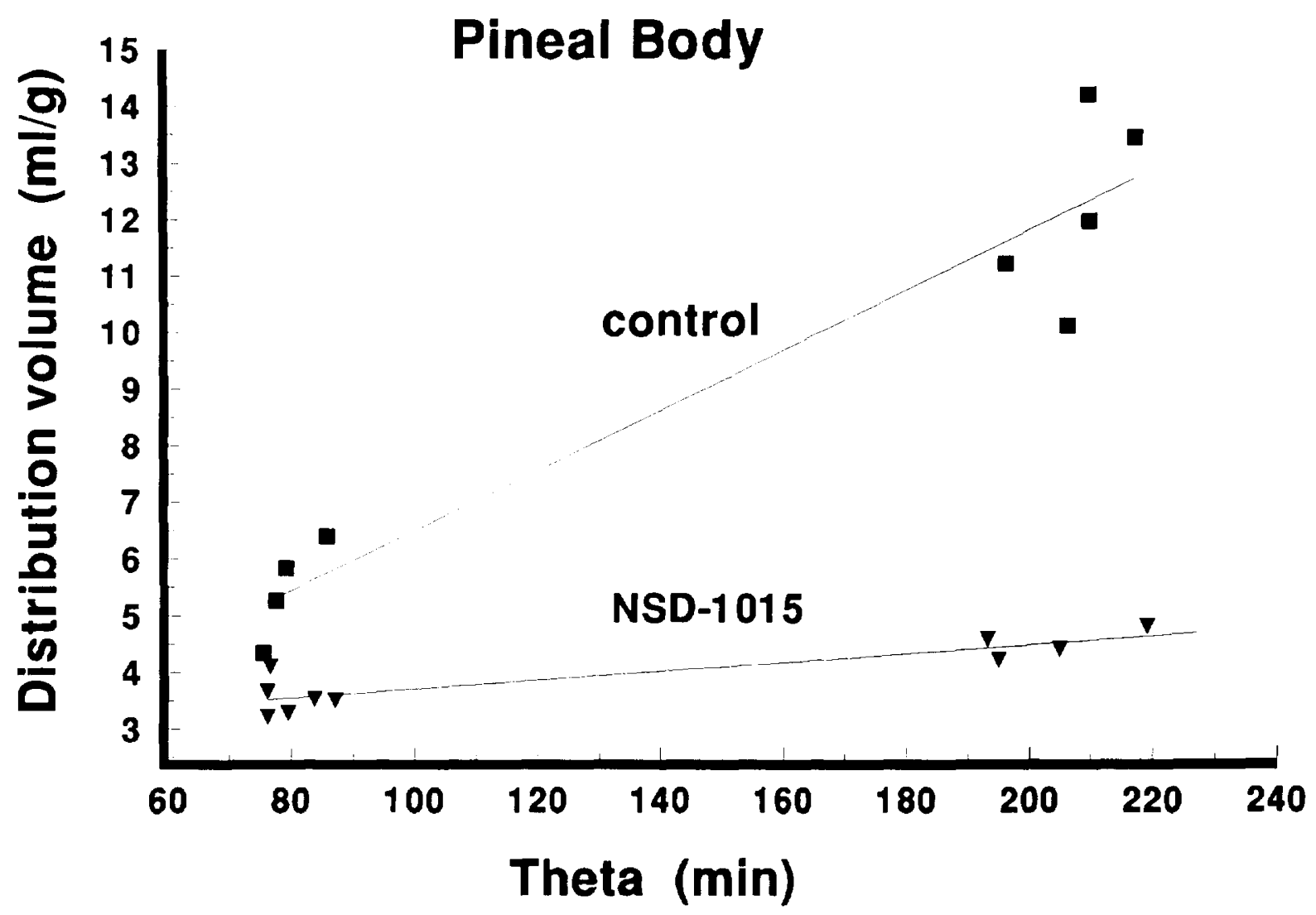

Figure 5. Tissue distribution volume $\left(\mathrm{ml} / \mathrm{g}^{-1}\right)$ of tracer for control $(\boldsymbol{\square})$ and NSD-1015-treated $(\mathbf{\Delta})$ rats killed at 60 and 150 minutes after tracer injection as a function of theta (minutes) in the pineal body.

our experimental period. The rate of 5 -HT synthesis reported here represents an average rate between 3 and $41 / 2$ hours after reserpine administration, but without any additional pharmacological treatment.
In order to investigate the possibility that the reduction in the synthesis rate might be related to a possible loss of the label from the tissue, we performed experiments to prove that reserpine by itself does not

Table 2. The Rate of 5 -HT Synthesis $\left(R ;\right.$ pmol $\mathrm{g}^{-1}$ minute $\left.^{-1}\right)$ in Representative Brain Structures and Pineal Body in Control and NSD-1015-Treated Rats

\begin{tabular}{lccc}
\hline & \multicolumn{3}{c}{$\mathbf{R}^{\boldsymbol{a}}\left(\mathbf{p m o l} \mathbf{g}^{-\mathbf{1}} \mathbf{m i n u t e}^{-\mathbf{1}} \mathbf{c}\right.$} \\
\cline { 2 - 4 } \multicolumn{1}{c}{ Structure } & $\begin{array}{c}\text { Control } \\
(\boldsymbol{n}=\mathbf{1 0})\end{array}$ & $\begin{array}{c}\text { NSD-1015 } \\
(\boldsymbol{n}=\mathbf{1 1})\end{array}$ & $\begin{array}{c}\text { Difference } \\
\mathbf{( \% )}^{\boldsymbol{b}}\end{array}$ \\
\hline Dorsal raphe nucleus & $220 \pm 32$ & $277 \pm 39$ & $26^{c}$ \\
Median raphe nucleus & $163 \pm 31$ & $184 \pm 19$ & 13 \\
Hypothalamus & $58 \pm 10$ & $85 \pm 15$ & $47^{d}$ \\
Visual cortex & $56 \pm 15$ & $66 \pm 17$ & 18 \\
Caudate nucleus lateral & $54 \pm 16$ & $81 \pm 19$ & $50^{c}$ \\
Caudate nucleus medial & $84 \pm 19$ & $111 \pm 24$ & $32^{e}$ \\
Substantia nigra pars reticulata & $34 \pm 8$ & $66 \pm 9$ & $94^{d}$ \\
Substantia nigra pars compacta & $53 \pm 20$ & $73 \pm 13$ & $38^{c}$ \\
Hippocampus ventral & $79 \pm 21$ & $102 \pm 16$ & $29^{c}$ \\
Globus pallidus & $54 \pm 10$ & $70 \pm 10$ & $30^{c}$ \\
Nucleus accumbens & $90 \pm 14$ & $137 \pm 27$ & $52^{d}$ \\
Pineal body & $879 \pm 101$ & $139 \pm 27$ & $84^{d}$ \\
\hline
\end{tabular}

\footnotetext{
${ }^{a}$ The rates are given as estimates $\pm \mathrm{SD} ; n$ is the number of animals.

${ }^{b}$ Differences are given as a percentage changes from the rate in the control group. The difference is significant when NSD-1015 group is compared to the control group.

${ }^{c} p<.002$ (two-tail $t$-test).

${ }_{d} p<.001$ (two-tail $t$-test).

e $p<.02$ (two-tail $t$-test).
} 


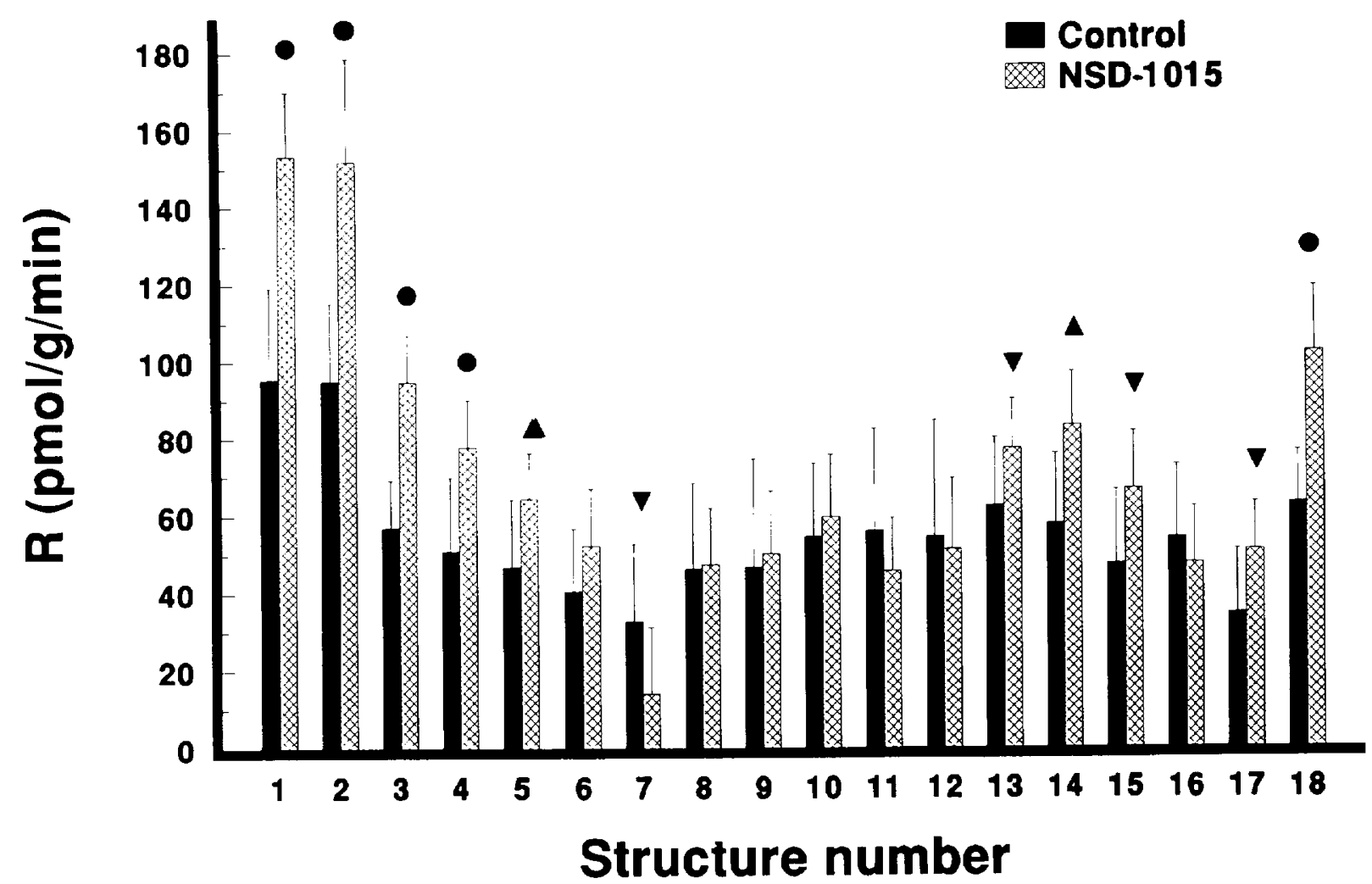

Figure 6. A comparison of the 5-HT synthesis rates $\left(R ; \mathrm{pmol} \mathrm{g}^{-1} \mathrm{~min}^{-1}\right)$ in some additional brain structures (not given in Table 2) in control (filled bars) and NSD-1015-treated (crossed bars) rats. Each column represents mean \pm SD (shown as error bars) of 10 (control) or 11 (NSD-1015-treated) animals. Structures number are: (1) raphe obscurus, (2) raphe pallidus, (3) raphe magnus, (4) ventral tegmental area, (5) medial forebrain bundle, (6) superior olive, (7) inferior colliculus, (8) superior colliculus, (9) median geniculate body, (10) lateral geniculate body, (11) ventral thalamus, (12) dorsal thalamus, (13) dorsal hippocampus, (14) auditory cortex, (15) parietal cortex, (16) sensory-motor cortex, (17) frontal cortex, (18) medial anterior olfactory nucleus. $\nabla p<.05 ; \Delta p<.02 ; \bullet p<.001$, as compared with the value in the control group.

influence the amount of the label in the tissue. These data showed that the labeled tracer and/or metabolite could not diffuse out of the brain after reserpine treatment (release of the label into cytoplasm), at least not during the time interval used in our present protocol (see above and Figure 4). These experiments were designed to determine whether there would be a loss of the label from the tissue (e.g., structure of interest) after the labeled compound was released by the reserpine into the cytoplasm. To test this we administered reserpine 100 minutes after the tracer injection, because at that time the tracer (at least a larger fraction of it) was trapped in the tissue-reversible compartment, from which we assume there were no losses of the label. An interval of 100 minutes between two injections and between injection of reserpine and sacrifice was selected to allow comparison with our experimental protocol, in which the serotonin synthesis rate was measured. Since the synthesis rate in our protocol was calculated from the slope of the DV as a function of exposure time $\Theta$, the greatest influence on the slope would come from the reduction of DV in rats killed 150 minutes after tracer injection (Equation 1). Statistical comparison of the DV in control and reserpine-treated rats at 100 minutes after tracer and killed 100 minutes after the injection of reserpine showed that there was no significant loss of the label from any of the structures examined (Figure 4). This is contrary to the observation of Carlsson and Waldeck (1968), who reported a loss of metaraminol from the heart of mice after administration of reserpine. However, both metaraminol and $\alpha$-MTrp are not substrates for the monoamine oxidase (MAO), but metaraminol is a greatly different compound and it was studied in a very different organ (heart). These data confirm that when this tracer was released into the cytoplasm from the vesicular storage with reserpine, there was no significant loss of the labeled tracer from the brain structure under evaluation (Figure 4). In other words, a loss of the label from the brain could not explain the decrease in the serotonin synthesis rate obtained with reserpine in the experiments reported here.

It is well-known that tryptophan shares a common carrier system with other LNAA for active transport across the BBB. Consequently, an increased plasma level of those amino acids should directly influence tryptophan uptake to the brain and also disturb the uptake 


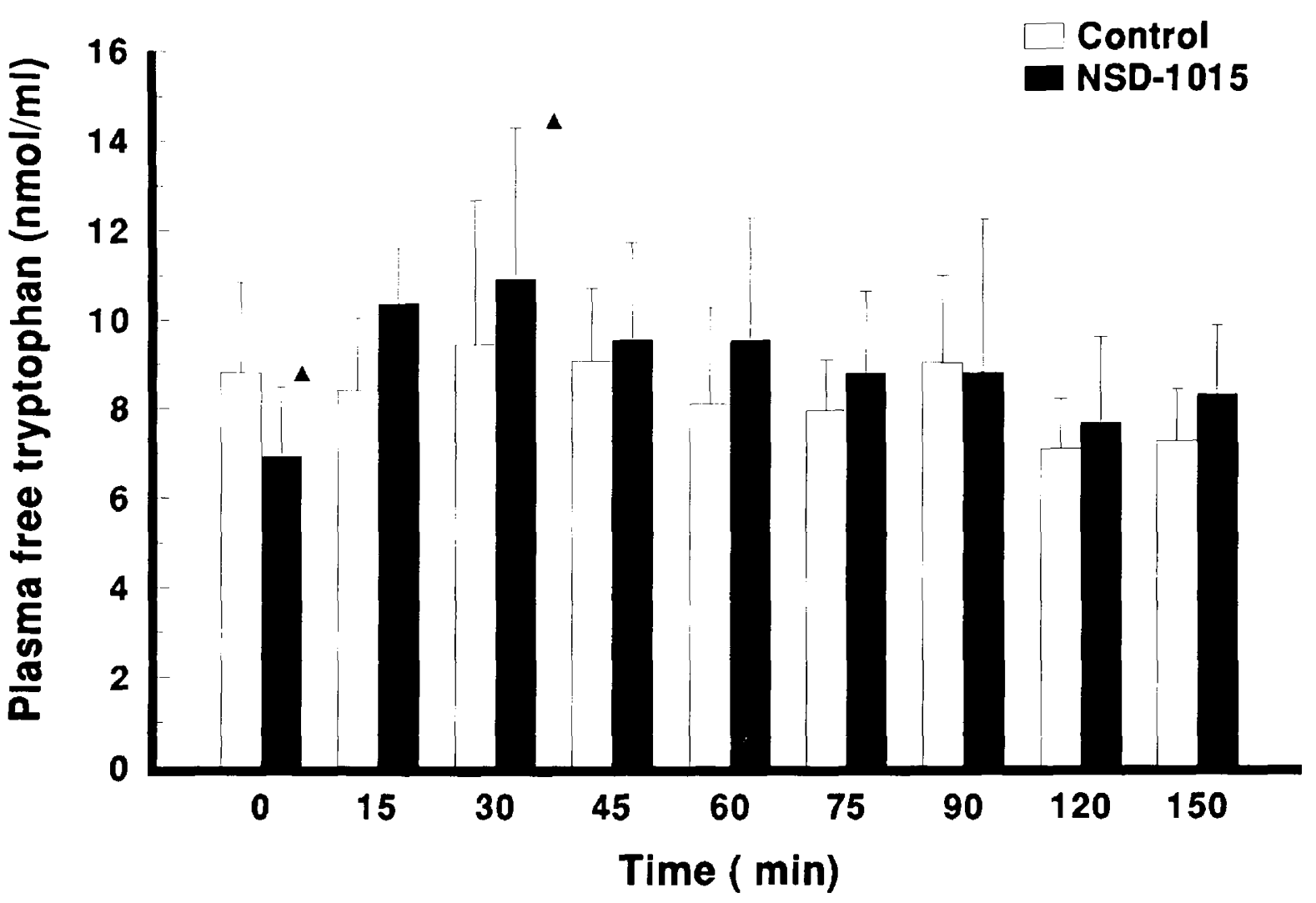

Figure 7. Time-dependent effect of NSD-1015 (100 mg/kg IP; filled bars) and control (saline; open bars) on plasma-free tryptophan concentration. Zero denotes the values for free tryptophan just before injection of NSD-1015 or saline. Each column represents mean \pm SD for seven to nine animals. $\Delta p<.05$ (ANOVA followed by Newman-Keuls's test).

of the other LNAA (Voog and Eriksson 1992). The rats used in the present work were deprived of food during the night before experiments, so that the concentration of LNAA in the rat plasma during the experiment was constant. Data showed that reserpine injection did not influence the plasma levels of free and total tryptophan, which is in agreement with the data of Long et al. (1983). Reserpine treatment did not have any effect on the concentrations of other LNAA. These results indicated that the decrease in 5-HT synthesis rate in reserpine-treated rats found in the present work could not be a consequence of a disturbance in the uptake of plasma tryptophan into the brain.

It was reported earlier that the increase in plasmafree tryptophan increases the brain 5-HT synthesis rate (Bloxam and Curzon 1978; Diksic et al. 1991). In the present work we found that NSD-1015 (a commonly used inhibitor of decarboxylase activity) induces a timedependent increase in the plasma-free tryptophan that could influence the transport of tryptophan from plasma into the brain and consequently increase 5-HT synthesis. Since NSD-1015 treatment increases plasmafree tryptophan levels, the increase in the 5-HT synthesis rate reported by Hjorth (1992) can be at least in part explained by the increase of the plasma-free tryptophan fraction rather than by the action of reserpine itself. Unfortunately, Hjorth (1992) did not measure the plasma concentration of amino acids that would permit us to normalize his data.

The observed decrease in the 5-HT synthesis rate after acute reserpine application could be explained by the change in the activity of TPH. Since it is known that this enzyme is not inhibited by 5-HT (reaction end product) (Joh et al. 1986), there is a possibility that reserpine has a direct or indirect influence on the TPH activity or its synthesis. The literature data concerning the direct effect of reserpine on TPH activity are confusing. It seems that the influence of reserpine on TPH activity (measured in vitro) is time-dependent since a decrease (Park et al. 1993) and an increase (Park et al. 1993; Živković et al. 1973) were observed 1 day or 4 days after reserpine application, respectively. However, there are no data about enzyme activity in the first few hours after reserpine treatment, although the data presented in this work (in vivo assessment of enzyme activity) suggest that reserpine probably inhibits the TPH activity via monoamines and/or monoamine metabolites. Since Park et al. (1993) reported a decrease in the TPH activ- 
ity 1 day after reserpine treatment, it is possible that the decrease observed by us was part of the decrease that occurs in acutely treated animals. Park et al. (1993) also reported that the reserpine treatment does not influence the steady-state concentration of the TPH mRNA. This suggests that a de novo synthesis of TPH is not influenced directly or indirectly by the reserpine treatment. Regardless of the constant levels of TPH enzyme, the synthesis of $5-\mathrm{HT}$ could be changed by an enzyme deactivation mechanism through reserpine's effect on the brain dopaminergic neurons. In brief, reserpine is a potent, but not selective, drug since it produces the release of brain 5-HT (Halaris and Freedman 1975; Long et al. 1983; Oishi et al. 1993), dopamine (DA; Oishi et al. 1993), and noradrenaline (Oishi et al. 1993; Ugedo et al. 1993) from synaptic vesicles, as well as histamine from neurons and nonneuronal mast cells elements (Russell et al. 1990). The released DA could be taken up into serotoninergic nerve endings (Waldmeier 1985) and could exert untoward effects on 5-HT synthesis via inhibition of TPH activity (Johansen et al. 1991).

Other explanations for the decrease of 5-HT synthesis after acute reserpine application could also be related to the change in activity of 5-HT receptors. Using microdialysis, it was shown that acute reserpine administration increases the extracellular concentration of 5-HT (Martin and Artigas 1992), which could stimulate 5-HT autoreceptors and decrease 5-HT synthesis through a feedback mechanism (Briley and Moret 1993). This is supported by the findings that acute (Tunnicliff et al. 1992) or local (Invernizzi et al. 1991) administration of buspirone (a 5- $\mathrm{HT}_{1 \mathrm{~A}}$ agonist) reduces $5-\mathrm{HT}$ synthesis. However, both investigations determined that 5-HT synthesis using 5-HTP accumulation after NSD1015 induced inhibition of decarboxylase activity. Since they report a decrease in the rate of 5-HT synthesis, but NSD-1015 actually increases synthesis (see earlier), the effect of buspirone is probably even greater.

In summary, we found a significant decrease in the 5 -HT synthesis rate in 26 out of 27 rat brain regions after acute (3-4.5 hours) reserpine application. This effect of reserpine could be explained through its direct or indirect influence on TPH activity. On the other hand, NSD-1015 alone induced the alterations in the 5-HT synthesis rate. On the basis of the present results, the meaning of the data on the 5-HT synthesis collected until now using NSD-1015 should be interpreted with caution.

\section{ACKNOWLEDGMENTS}

The work was supported in part by the U.S. Public Service (ROI N29629) and the Medical Research Council of Canada (MA-10232). The authors would like to thank Mrs. Janet Arts for technical assistance and Ms. Carol Comfort for help in preparing the manuscript.

\section{REFERENCES}

Bloxam DL, Curzon G (1978): A study of proposed determinants of brain tryptophan concentration in rats after portocaval anastomoses or sham operation. J Neurochem 31:1255-1263

Briley M, Moret C (1993): Neurobiological mechanisms involved in antidepressant therapies. Clinical Neuropharmacol 16:387-400

Byerly WF, Risch C (1985): Depression and serotonin metabolism rationale for neurotransmitter precursor treatment. J Clin Psychopharmacol 5:191-206

Carlsson A, Waldeck B (1968): Different mechanisms of druginduced release of noradrenaline and its congeners $\alpha$-methylboradrenaline and metaraminol. Eur J Pharm 4:165-168

Diksic M, Nagahiro S, Sourkes TL, Yamamoto YL (1990): A new method to measure brain serotonin synthesis in vivo. I. Theory and basic data for a biological model. J Cereb Blood Flow Metab 10:1-12

Diksic M, Nagahiro S, Chaly T, Sourkes TL, Yamamoto YL, Feindel W (1991): Serotonin synthesis rate measured in living dog brain by positron emission tomography. J Neurochem 56:153-162

Halaris AE, Freedman DX (1975): Loss of body weight as a predictor of reserpine-induced amine depletion. Eur J Pharmacol 32:93-101

Hjorth S (1992): Acute reserpine treatment increases rat brain serotonin synthesis via a nerve impulse-dependent mechanism. J Neurochem 58:772-775

Hrdina PD, Demeter E, Vu TB, Sotonyi P, Palkovits M (1993): 5 -HT uptake sites and $5-\mathrm{HT}_{2}$ receptors in brain of antidepressant-free suicide victims/depressives: Increase in $5-\mathrm{HT}_{2}$ sites in cortex and amygdala. Brain Res $614: 37-44$

Invernizzi R, Carli M, Di Clemente A, Samanin R(1991): Administration of 8-Hydroxy-2-(di-n-propylamino)tetralin in raphe nuclei dorsalis and medianus reduces serotonin synthesis in the rat brain: Differences in potency and regional sensitivity. J Neurochem 56:243-247

Joh TH, Hwang O, Abate C (1986): Phenylalanine hydroxylase, tyrosine hydroxylase and tryptophan hydroxylase. In Boulton AA, Baker GB, Yu PH (eds), Neuromethods Series 1: Neurochemistry, Neurotransmitter Enzymes, Clifton, NJ: Humana Press, pp 1-32

Johansen P, Wolf WA, Kuhn DM (1991): Inhibition of tryptophan hydroxylase by benserazide and other catechols. Biochem Pharmacol 41:625-628

Joyce JN (1993): The dopamine hypothesis of schizophrenia: Limbic interactions with serotonin and norepinephrine. Psychopharmacol 112:S16-S34

Kleven MS, Dwoskin LP, Sparber SB (1983): Pharmacological evidence of multiple functional pools of brain serotonin: Analysis of brain perfusate from conscious rats. J Neurochem 41:1143-1149

Kuhar MJ, Roth RH, Aghajania GK (1971): Selective reduction of tryptophan hydroxylase activity in rat forebrain after midbrain raphe lesions. Brain Res 35:167-176

Kuhn DM, Wolf WA, Youdim BH (1985): 5-Hydroxytryptamine release in vivo from a cytoplasmatic pool: Studies 
on the 5-HT behavioural syndrome in reserpinized rats. Br J Pharmacol 84:121-129

Long JB, Youngblood WY, Kizer JS (1983): Regional differences in the response of serotoninergic neurons in rat CNS to drugs. Eur J Pharmacol 88:89-97

Martin F, Artigas F (1992): Simultaneous effects of p-chloroamphetamine, d-fenfluramine and reserpine on free and stored 5-Hydroxytryptamine in brain and blood. J Neurochem 59:1138-1144

Mzengeza S, Venkatachalam TK, Rajagopal S, Diksic M (1993): Synthesis of enantiomerically pure $\alpha-\left[{ }^{14} \mathrm{C}\right]$ methyl-Ltryptophan. Appl Radiat Isot 44(9):1167-1172

Nagahiro S, Takada A, Diksic M, Sourkes TL, Missala K, Yamamoto YL (1990): A new method to measure brain serotonin synthesis in vivo. II. A practical autoradiographic method tested in normal and lithium-treated rats. J Cereb Blood Flow Metab 10:13-21

Oishi R, Suemaru K, Furuno K, Gomita Y, Saeki K (1993): Possible explanation for the antagonism by nicotine against reserpine-induced depletion of monoamines in mouse brain. Naunyn-Schmiedeberg's Arch Pharmacol 348:154-157

Park DH, Wessel TC, Joh TH (1993): Acute effects of reserpine on tryptophan hydroxylase activity and mRNA in rat brain. Brain Res 620:331-334

Rudnick G, Clark J (1993): From synapse to vesicles: The reuptake and storage of biogenic amine neurotransmitters. Biochem Biophys Acta 1144:249-263

Russell WL, Henry DP, Phebus LA, Clemens JA (1990): Release of histamine in rat hypothalamus and corpus striatum in vivo. Brain Res 512:95-101
Schuldiner S, Liu Y, Edwards RH (1993): Reserpine binding to a vesicular amine transporter expressed in Chinese hamster ovary fibroblasts. J Biol Chem 268:29-34

Takada A, Grdiša M, Diksic M, Gjedde A, Yamamoto YL (1993): Rapid steady-state analysis of blood-brain transfer of L-Trp in rat, with special reference to the plasma protein binding. Neurochem Int 23:351-359

Tunnicliff G, Brokaw JJ, Hausz JA, Matheson GK, White GW (1992): Influence of repeated treatment with buspirone on central 5-hydroxytryptamine and dopamine synthesis. Neuropharmacol 31:991-995

Ugedo L, Garro MA, Pineda J, Giralt MT, Miralles A, Olmos G, Garcia-Sevilla JA, Menargues A, Obach R (1993): Acute and chronic effects of reserpine on biochemical and functional parameters of central and peripheral $\alpha_{2^{-}}$ adrenoceptors. Eur J Pharmacol 239:149-157

Vanier M, Tsuiki K, Grdiša M, Worsley K, Diksic M (1995): Determination of the lumped constant for the $\alpha$-methyltryptophan method of estimating the rate of serotonin synthesis. J Neurochem 64:624-635

Voog L, Eriksson T (1992): Relationship between plasma and brain large neutral amino acids in rats fed diets with different compositions at different times of the day. J Neurochem 59:1868-1874

Waldmeier PC (1985): Displacement of striatal 5-hydroxytryptamine by dopamine released from endogenous stores. J Pharm Pharmacol 37:58-60

Živković B, Guidotti A, Costa E (1973): Increase of tryptophan activity elicited by reserpine. Brain Res 57:522-526 